DOI: $10.5216 /$ cab.v13i3.17565

\title{
AVALIAÇÃO DAS DIETAS SIMPLIFICADAS E SEMI- SIMPLIFICADAS, COM BASE NA MISTURA DE FORRAGEIRAS, COM E SEM ENZIMAS, PARA COELHOS EM CRESCIMENTO
}

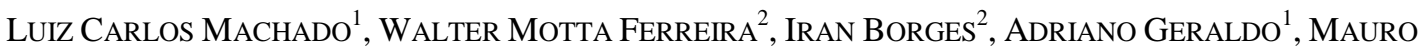 \\ FERREIRA $^{3}$
}

${ }^{1}$ Professor Doutor do Instituto Federal Minas Gerais, Bambui, MG - Brasil - luiz.machado@ifmg.edu.br

${ }^{2}$ Professores Doutores da Universidade Federal de Minas Gerais, Belo Horiznte, MG, Brasil.

${ }^{3}$ Zootecnista Autônomo, Bambuí, MG, Brasil.

\begin{abstract}
As dietas simplificadas e semi-simplificadas para coelhos são uma nova tecnologia que associa economia e bem estar intestinal. O objetivo principal deste trabalho foi avaliar o efeito das dietas simplificadas e semisimplificadas, com e sem enzimas exógenas, sobre o desempenho produtivo de coelhos em crescimento. Foram utilizadas 11 dietas, sendo uma referência, duas simplificadas e oito semi-simplificadas, formuladas, contendo como principais fontes fibrosas o feno de alfafa (FAL), feno do terço superior da rama da mandioca (FTSRM) e ou a farinha das folhas de mandioca (FFM), com ou sem adição de enzimas exógenas Os animais foram distribuídos em um delineamento inteiramente cazualizado com 11 tratamentos e oito repetições. As enzimas utilizadas foram as carboidrases (alfa-
\end{abstract}

galactosidase, galactomanase, xilanase e betaglucanase) e a fitase. O consumo dos animais que receberam as dietas com elevada inclusão de FFM foi inferior ao demais. O ganho de peso diário mais elevado foi observado com os animais alimentados com as dietas referência, semisimplificada com base em FTSRM e semi-simplificada com base na mistura de FAL e FFM. Não foram observados efeitos positivos das enzimas exógenas sobre os parâmetros de desempenho. Com base nestes resultados conclui-se que as dietas simplificadas e semisimplificadas proporcionam queda no desempenho produtivo. Considerando-se a economia proporcionada no custo da alimentação, a dieta semi-simplificada com base em FTSRM pode ser uma alternativa interessante.

PALAVRAS-CHAVE: cunicultura, desempenho produtivo, rama da mandioca.

\section{EVALUATION OF SIMPLIFIED AND HALF-SIMPLIFIED DIETS ON THE BASIS OF THE MIXTURE OF FORAGE CROPS, WITH AND WITHOUT ENZYMES, FOR GROWING RABBITS}

\section{ABSTRACT}

The simplified and half-simplified diets are a new technology that combines economy and welfare intestinal. The main objective of this study was to evaluate the effect of simplified and half-simplified diets, with and without exogenous enzymes, on the productive performance of growing rabbits. A Reference diet, two simplified diets and eight half-simplified diets had been utilized. The main fibrous sources used were the alfalfa hay (ALH), hay from the upper third of the cassava foliage (HUTCF) and cassava leaf meal (CLM), using a completely randomized design with 11 treatments and eight repetitions. The enzymes used were the carbohydrases (alphagalactosidase, galactomanase, xylanase and betaglucanase) and phytase. The consumption of animals fed with diets with high inclusion of CLM was lower than the other. The daily weight gain was higher in animals fed with the reference diet, half-simplified diet based on HUTCF and half-simplified diets based on the mix of ALH and CLM. A positive effect of exogenous enzymes inclusion, on the performance parameters, was not 
observed. Based on these results we conclude that the simplified and half-simplified diets provide decrease in productive performance. Considering the proportionate economy in the cost of feed, the half simplified based on HUTCF may be an interesting alternative.

KEYWORDS: cassava foliage, productive performance, rabbit production.

\section{INTRODUÇÃO}

A cunicultura é uma atividade estratégica dos pontos de vista econômico, social e ambiental, pois o coelho é altamente prolífero, produtivo, possui carne de excelente qualidade, adequa-se às pequenas propriedades, aceita dietas com grande quantidade de ingredientes fibrosos e causa baixo impacto ambiental, quesitos fundamentais para o desenvolvimento sustentável da sociedade moderna. Para sucesso dessa atividade, é essencial a pesquisa por formas alternativas de alimentação a baixo custo.

Nesse sentido, destacam-se as dietas com alta inclusão de alimentos forrageiros, também denominadas de simplificadas. O estudo dessas dietas para coelhos vem sendo conduzido por vários autores (FERNANDEZ-CARMONA et al., 1998; PASCUAL et al., 2002; FERREIRA et al., 2006; MACHADO et al., 2007; FARIA et al., 2008; OLIVEIRA et al., 2011), sendo contraditórios os resultados obtidos até o momento. De maneira geral, há redução da incidência de diarreias, ocasionada pela melhora na saúde intestinal, além de redução no desempenho animal em relação ao obtido com a utilização de dietas formuladas com ingredientes tradicionais.

A fim de buscar o ponto de equilíbrio entre a máxima economia e o desempenho satisfatório do animal, MACHADO et al. (2007) sugeriram a utilização de dietas semi-simplificadas, adicionando pequena quantidade de fontes de amido e proteína de boa qualidade nutricional, para melhoria geral do processo digestivo, principalmente da atividade fermentativa. Outra sugestão feita pelos autores, e posteriormente indicada por FARIA et al. (2008), é a mistura de diferentes forrageiras, visando complementaridade e melhoria da eficiência de utilização pelos animais. Atualmente, grande parte das rações fornecidas aos animais monogástricos recebe adição de enzimas exógenas que melhoram o processo digestivo, incrementando o aproveitamento dos nutrientes e reduzindo o efeito antinutricional causado por algumas substâncias.

Este trabalho teve como objetivo avaliar o desempenho produtivo de coelhos em crescimento recebendo dietas simplificadas e semi-simplificadas com base em uma forrageira ou na mistura entre elas, com e sem adição de enzimas fitase e carboidrases.

\section{MATERIAL E MÉTODOS}

O experimento foi conduzido no laboratório de metabolismo animal, do departamento de Zootecnia da EV-UFMG, no período de 07 de Dezembro de 2009 a 16 de Janeiro de 2010. Durante o experimento, as temperaturas mínima e máxima médias foram de 20,8 e $26,7^{\circ} \mathrm{C}$, respectivamente, e a média geral foi de $23,7^{\circ} \mathrm{C}$. As gaiolas utilizadas foram de arame galvanizado, de tamanho $0,30 \times 0,60 \mathrm{~m}$, numa área disponível de $0,18 \mathrm{~m}^{2}$. Anteriormente à chegada dos animais, as instalações foram limpas e desinfetadas com auxílio de vassoura de fogo. O bebedouro utilizado foi o do tipo chupeta automático e o comedouro semi-automático.

Os animais utilizados foram provenientes da fazenda experimental Prof. Hélio Barbosa, localizada no município de Igarapé-MG, e consistiram de coelhos da raça Nova Zelândia Branca, de ambos os sexos, desmamados aos 35 dias de idade. Ao início do experimento, o peso inicial foi equilibrado entre os tratamentos. Foram utilizados oito animais por dieta experimental, num total de 88 animais. A aprovação do comitê de ética foi registrada como $171 / 08$ do CETEA/UFMG.

Os tratamentos, os quais são detalhados na Tabela 1, consistiram das seguintes dietas: REF - dieta referência; SFA - dieta simplificada com base na mistura de farinha das folhas de mandioca (FFM) e feno de alfafa (FAL); SSM - dieta semi-simplificada com base em feno do terço superior da rama da mandioca (FTSRM); SSA - dieta semi-simplificada com base em FAL; SSF - dieta semi-simplificada com base em FFM; SSFA - dieta semi-simplificada com base na mistura de FFM e FAL; SSMA - dieta semi-simplificada com base na mistura de FTSRM e FAL; SFAE - dieta SFA + enzimas carboidrases e fitase; SSME - dieta SSM + enzimas carboidrases e fitase - SSFE: dieta SSF + enzimas carboidrases e fitase e SSFAE - dieta SSFA + enzimas carboidrases e fitase. A dieta referência foi formulada para atender às exigências propostas por DE BLAS \& MATEOS (1998) e as dietas simplificadas e semi-simplificadas foram formuladas objetivando-se, ao máximo, a aproximação das exigências citadas acima e, quando não foi possível ajustar o conteúdo de $\mathrm{ED}$, procurouse fornecer o mínimo de $2200 \mathrm{kcal} / \mathrm{kg}$, como proposto por CHEEKE (1994), pois os coelhos conseguem regular o seu consumo em função da densidade energética da ração.

Foram verificados os níveis dos aminoácidos lisina, metionina + cistina, treonina, triptofano e arginina, embora não tenha sido necessária a adição dos três últimos. Para incremento do conteúdo energético da dieta e melhoria na digestibilidade dos 
O desempenho produtivo dos animais foi avaliado pelos seguintes parâmetros: peso aos 75 dias (P75), consumo diário de ração (CDR), ganho de peso diário (GPD), conversão alimentar (CA) e mortalidade. Também foram avaliados o consumo diário de energia digestível (kcal/dia) e de proteína digestível (g/dia). Todos os animais foram pesados ao serem alojados nas gaiolas (início) e aos 75 dias de idade, por ocasião do abate (final do experimento).

Para cálculo do custo da ração necessária para produção de $1,0 \mathrm{~kg}$ de peso vivo, foi utilizada a CA geral de cada tratamento e foram tomados os preços de compra da fazenda experimental prof. Hélio Barbosa. O preço da FFM foi obtido diretamente na COOPATAN e o custo do FTSRM foi obtido a partir de FERREIRA et al. (2011).

$\mathrm{O}$ delineamento experimental foi inteiramente ao acaso sendo 11 tratamentos e oito repetições. Cada unidade experimental foi constituída de um coelho. As médias obtidas foram submetidas à análise de variância e comparadas pelo teste SNK a 5\% de probabilidade, utilizando-se os recursos do programa computacional SAS. Para os parâmetros P75, CDR e GPD, o peso aos 35 dias foi considerado como covariável.

\section{RESULTADOS E DISCUSSÃO}

Durante $o$ experimento não foram observadas diarreias ou quaisquer enfermidades digestivas. Não houve morte de animais. FERNANDEZ-CARMONA et al. (1998) relataram $11 \%$ de mortalidade durante o experimento, em que o tratamento referência apresentou os piores resultados quando comparado às dietas simplificadas. Esse efeito foi também observado por HARRIS et al. (1981), que perceberam redução da incidência de diarreia e enterite com a elevação dos níveis de fibra.

O consumo dos animais foi influenciado pela dieta $(p<0,05)$. Os tratamentos SSF, SFAE e SSFE foram os que proporcionaram os valores mais baixos, provavelmente devido à baixa palatabilidade da FFM. FERREIRA et al. (2006) trabalharam com dietas simplificadas e verificaram consumo de 88,9 e $84,3 \mathrm{~g}$ para as dietas simplificadas com base em FAL e referência, sendo esses valores inferiores aos aqui observados. Já considerando a dieta simplificada com base em FTSRM, o consumo de ração foi mais elevado quando comparado a esse experimento. HARRIS et al. (1981) observaram elevação no consumo à medida que se elevaram os níveis de fibra dietética, o que não foi observado neste experimento. OLIVEIRA et al. (2011) testaram dieta semisimplificada com $81,63 \%$ de subprodutos da mandioca e observaram queda no consumo em comparação com a dieta referência, sendo os valores mais elevados do que os deste experimento. Situação semelhante é observada quando os resultados são comparados a MACHADO et al. (2006), que perceberam consumo de ração mais elevado quando comparados aos valores aqui verificados. Vários são os fatores que podem ter contribuído para menor consumo neste experimento, dentre eles pode-se destacar os baixos níveis de componentes fibrosos (GIDENNE, 2000), palatabilidade das rações, diferenças ambientais, principalmente em relação à temperatura, e diferenças genéticas entre os diferentes grupos de animais considerados.

Deve-se chamar atenção para a temperatura média no período do experimento, realizado em condições de primavera-verão. A temperatura mínima média foi de $20,8^{\circ} \mathrm{C}$ e a máxima média foi de $26,7^{\circ} \mathrm{C}$, sendo a média geral de $23,7^{\circ} \mathrm{C}$, superior à zona de conforto térmico para coelhos (MULLER, 1982). Altas temperaturas proporcionam inibição do consumo pelos animais e podem explicar parte dos motivos que proporcionaram baixo consumo neste experimento em comparação a outros. MACHADO et. Al. (2006) trabalharam numa temperatura média de $19,2^{\circ} \mathrm{C}$.

Houve influência das dietas experimentais sobre o ganho de peso diário $(\mathrm{p}<0,05)$. Os tratamentos SSM e SSFA apresentaram resultados semelhantes à dieta REF. Os demais tratamentos apresentaram resultados inferiores. Os piores resultados foram observados nos tratamentos SSF, SSFE, SFA e SFAE, que apresentavam elevada inclusão de FFM. Valores intermediários de ganho de peso diários foram observados para os tratamentos SSA, SSMA, SSME, SSFAE.

Trabalhando com dietas simplificadas com base em FAL, FERNANDEZ-CARMONA et al. (1998) obtiveram GDP médio de 37,25 g/dia, sendo esses valores superiores a todos os observados neste trabalho, inclusive ao tratamento referência. O elevado potencial genético dos animais europeus pode ter sido o principal fator de contribuição para esse fato. $\mathrm{O}$ peso médio dos coelhos aos 35 dias utilizado neste experimento foi de $719 \mathrm{~g}$, enquanto o peso na mesma idade, relatado por aqueles autores, foi de $853 \mathrm{~g}$, o que reforça a ideia de maior potencial genético.

Outro fator que merece destaque é a temperatura ambiental relatada, que foi de 12 a $19^{\circ}$ $\mathrm{C}$, próxima à faixa de conforto térmico do animal (MULLER, 1982). Nessa faixa, há elevação no CDR e o GPD é otimizado, havendo a minimização do incremento calórico ou demais perdas advindas da tentativa de reversão do estresse calórico. Nas 
condições brasileiras de primavera-verão, quando se realizou este experimento, as temperaturas médias foram de 20,8 a $26,7^{\circ} \mathrm{C}$ para a mínima e máxima, respectivamente, e, como já comentado, o consumo foi prejudicado.

Tabela 02 - Peso vivo aos 75 dias (P75), ganho de peso diário (GPD), consumo diário de ração (CDR), conversão alimentar (CA) de coelhos, de acordo com as dietas experimentais

\begin{tabular}{lcccc}
\hline & & & Parâmetros & \\
\hline Dietas & P75 $(\mathrm{g})$ & GPD $(\mathrm{g} / \mathrm{dia})$ & CDR $(\mathrm{g} / \mathrm{dia})$ & CA \\
REF & $2159,75 \mathrm{a}$ & $35,60 \mathrm{a}$ & $105,79 \mathrm{a}$ & $2,98 \mathrm{a}$ \\
SFA & $1754,38 \mathrm{bc}$ & $25,81 \mathrm{cde}$ & $96,19 \mathrm{ab}$ & $3,76 \mathrm{bc}$ \\
SSM & $1924,00 \mathrm{~b}$ & $31,66 \mathrm{ab}$ & $100,09 \mathrm{ab}$ & $3,20 \mathrm{ab}$ \\
SSA & $1914,86 \mathrm{~b}$ & $29,23 \mathrm{bcd}$ & $101,66 \mathrm{ab}$ & $3,50 \mathrm{abc}$ \\
SSF & $1623,43 \mathrm{c}$ & $21,52 \mathrm{e}$ & $85,70 \mathrm{bc}$ & $4,06 \mathrm{c}$ \\
SSFA & $1933,63 \mathrm{~b}$ & $31,53 \mathrm{ab}$ & $100,80 \mathrm{ab}$ & $3,21 \mathrm{ab}$ \\
SSMA & $1898,13 \mathrm{~b}$ & $29,83 \mathrm{bc}$ & $98,35 \mathrm{ab}$ & $3,30 \mathrm{ab}$ \\
SFAE & $1682,50 \mathrm{c}$ & $24,20 \mathrm{de}$ & $87,69 \mathrm{bc}$ & $3,69 \mathrm{bc}$ \\
SSME & $1878,25 \mathrm{~b}$ & $29,62 \mathrm{bc}$ & $99,19 \mathrm{ab}$ & $3,37 \mathrm{ab}$ \\
SSFE & $1625,63 \mathrm{c}$ & $23,01 \mathrm{e}$ & $77,22 \mathrm{c}$ & $3,42 \mathrm{ab}$ \\
SSFAE & $1905,50 \mathrm{~b}$ & $28,43 \mathrm{bcd}$ & $99,04 \mathrm{ab}$ & $3,48 \mathrm{abc}$ \\
CV $(\%)$ & 8,22 & 13,42 & 10,49 & 11,97 \\
\hline
\end{tabular}

Médias com letras iguais na mesma coluna são iguais pelo teste SNK ao nível de 5\% de probabilidade

*Valores determinados após experimento de digestibilidade

REF - dieta referência; SFA - dieta simplificada com base na mistura de farinha das folhas de mandioca (FFM) e feno de alfafa (FAL); SSM - dieta semi-simplificada com base em feno do terço superior da rama da mandioca (FTSRM); SSA - dieta semi-simplificada com base em FAL; SSF - dieta semi-simplificada com base em FFM; SSFA - dieta semi-simplificada com base na mistura de FFM e FAL; SSMA - dieta semi-simplificada com base na mistura de FTSRM e FAL; SFAE - dieta SFA + enzimas carboidrases e fitase; SSME dieta SSM + enzimas carboidrases e fitase - SSFE: dieta SSF + enzimas carboidrases e fitase; e SSFAE - dieta SSFA + enzimas carboidrases e fitase.

HARRIS et al. (1981) também verificaram valores de GPD superiores aos aqui observados. FERREIRA et al. (2006) perceberam que as dietas referência, simplificada FAL e simplificada FTSRM proporcionaram ganhos de, respectivamente, 29,06; 19,09 e 23,40g/dia, sendo inferiores aos obtidos neste experimento. Os resultados também foram superiores aos observados por ABD EL-BAKI et al. (1993) que verificaram GPD de 29 e 20 g para a dieta referência e dieta com $45 \%$ de produtos da mandioca, respectivamente.

Utilizando dietas semi-simplificadas, FARIA et al. (2008) conseguiram GPD na ordem de 44,0, 37,0 e 35,0g/dia, utilizando dietas referência, simplificada FAL e simplificada FTSRM, sendo esses valores bastante elevados se comparados aos achados nestes experimento. Contudo, o consumo relatado pelos autores foi bem superior aos aqui relatados. Situação semelhante foi relatada por OLIVEIRA et al. (2011), que obtiveram GPD na ordem de 36,8 $\mathrm{g} / \mathrm{dia}$, superior ao valor observado neste experimento, principalmente quando comparado às dietas semi-simplificadas e simplificadas. Já considerando os valores relatados por MACHADO et al. (2006), os dados aqui obtidos para as dietas referência e simplificada com base em FAL, são semelhantes. Considerando a dieta simplificada com base em FTSRM, por outro lado, os valores aqui obtidos são superiores. Naquela situação, os autores relataram a baixa qualidade nutricional do FTSRM utilizado e enfatizaram a importância de novas pesquisas com cultivares de mandioca mais propícias para utilização na alimentação animal.

Em relação à conversão alimentar, verificase que houve semelhança entre o tratamento REF com os tratamentos SSM, SSA, SSFA, SSMA, SSME, SSFE e SSFAE, para todo o período experimental $(p>0,05)$. Os tratamentos SFA, SSF e SFAE apresentaram conversão alimentar inferior ao tratamento REF. Excetuando-se o tratamento SSF, todas as dietas apresentaram resultados similares aos obtidos por FERNANDEZ-CARMONA et al. (1998), que trabalharam com dietas simplificadas com base em feno de alfafa. Os resultados aqui obtidos foram melhores que os encontrados por FERREIRA et al. (2006), que verificaram valores de 4,84 a 5,08 de CA para as dietas simplificadas baseadas em FTSRM e FAL, respectivamente. Os valores são semelhantes aos relatados por HARRIS 
et al. (1981), que ainda observaram que quanto maior o teor de fibra da dieta simplificada, maior tende a ser a conversão alimentar. Os valores também são semelhantes aos observados por ABD EL BAKI et al. (1993). A CA das dietas semi-simplificadas, observada neste experimento, mostra-se melhor quando comparada aos achados de FARIA et al. (2008), que observaram CA de 4,0 e 4,6 para as dietas simplificadas FAL e FTSRM. OLIVEIRA et al. (2011) obtiveram CA de 3,16 para a dieta semisimplificada com subprodutos da mandioca, estando esse valor próximo aos observados neste experimento. No experimento de MACHADO et al. (2006), a CA alimentar, para a dieta baseada em FAL, foi de 3,88, valor superior ao observado para as dietas semi-simplificadas deste experimento. Supõese que dietas semi-simplificadas são mais bem aproveitadas quando comparadas às simplificadas. Nota-se que as conversões alimentares aqui observadas estão, de forma geral, mais baixas que as observadas em outros experimentos. Deve-se lembrar que o consumo observado foi também menor.

O P75 dos animais que receberam a dieta REF foi superior aos demais. Pesos finais intermediários foram observados nos animais que receberam as dietas SSM, SSA, SSFA, SSMA, SSME, SSFAE e SFA, sendo este último semelhante $(\mathrm{p}>0,05)$ aos tratamentos SSF, SSFE e SFAE, que apresentaram os piores resultados. Os resultados de P75 deste experimento são inferiores aos obtidos por FERNANDEZ-CARMONA et al. (1998). Conforme comentado, as distintas condições experimentais podem ter influenciado nessa diferença. OLIVEIRA et al. (2011) também verificaram valor de peso final mais elevado que os aqui observados, embora os autores também tenham percebido queda no desempenho a partir da administração de dietas simplificadas. Os valores são semelhantes aos relatados por FARIA et al. (2008), que trabalharam com dietas simplificadas. Já quando se comparam os valores aos achados de MACHADO et al. (2006), verifica-se que os aqui encontrados são superiores, principalmente quando se considera a dieta simplificada com base em FTSRM.

Os piores resultados de desempenho, em geral, podem ser atribuídos aos tratamentos SFA, SSF, SFAE e SSFE. Essas dietas, provavelmente, proporcionam menor eficiência digestiva, sendo essa prejudicada por taninos livres e condensados, conforme proposto por SCAPINELLO et al. (1999), pela complexação aminoácido-carboidrato, proporcionada pela alta temperatura de processamento da FFM (acima de $100^{\circ} \mathrm{C}$ ), ou ainda pelo baixo nível de amido (tratamentos SFA e SFAE), essencial, em quantidades mínimas, para melhor funcionamento do ceco.

A adição de enzimas não influenciou ( $>>0,05)$ nenhum dos parâmetros de desempenho em todas as comparações.

VALENTE et al. (2000) perceberam melhorias na CA proporcionada por uma ração que continha $0,05 \%$ de complexo enzimático, composto por protease e celulase. Outros parâmetros de desempenho não foram afetados pela inclusão das enzimas. GUO-XIAN et al. (2004) observaram que o GPD foi melhorado $(\mathrm{p}<0,05)$ com a inclusão de fitase, com o nível de $800 \mathrm{FTU} / \mathrm{kg}$ apresentando os melhores resultados, sendo a atividade enzimática da fitase superior à utilizada neste experimento, que foi de $500 \mathrm{FTU} / \mathrm{kg}$. Contudo, nenhuma diferença foi observada no CDR e na CA. O efeito positivo da fitase foi relatado também por EIBEN et al. (2008), que não perceberam diferenças no desempenho produtivo, quando o nível de fósforo foi reduzido de 0,58 a $0,35 \%$ com inclusão de $1000 \mathrm{FTU} / \mathrm{kg}$ de fitase, verificando assim o efeito valorizador da enzima para disponibilização de fósforo. Já FALCÃO \& CUNHA et al. (2008) perceberam que a inclusão de enzima carboidrase (galactosidade) não melhorou o desempenho produtivo de coelhos em crescimento.

Avaliando-se o CDR e a conversão alimentar, pode-se perceber que o $\mathrm{kg}$ de animal produzido foi de $\mathrm{R} \$ 1,97 ; 3,65 ; 1,89 ; 3,75 ; 3,13$; 2,$95 ; 3,06 ; 3,62 ; 2,02 ; 2,67$ e 3,34, respectivamente, para os tratamentos REF, SFA, SSM, SSA, SSF, SSFA, SSMA, SFAE, SSME, SSFE e SSFAE. Notase que a dieta simplificada com base em FTSRM é uma alternativa viável, proporcionando economia de $4 \%$ sobre o custo necessário para produção de $1 \mathrm{~kg}$ de coelho vivo, considerando o tratamento REF, o que concorda com os achados de FERREIRA et al. (2006). É necessário que se invista em sistemas de colheita mecanizada para maior aproveitamento deste importantíssimo recurso para alimentação animal, o qual não compete com a alimentação humana e pode ser utilizado também por famílias de baixa renda em situações de agricultura familiar.

\section{CONCLUSÕES}

As dietas simplificadas e semi-simplificadas proporcionam em geral desempenho inferior quando comparadas à dieta referência. A dieta semisimplificada com base em feno do terço superior da rama da mandioca é uma alternativa economicamente interessante.

As enzimas exógenas não proporcionam melhorias no desempenho dos animais.

\section{REFERÊNCIAS}


ABD EL-BAKI, S. M. A.; NOWAR, M. S.; BASSUNY, S. M.; HASSONA E. M; SOLIMA E. S. Cassava as new animal feed in Egypt 3-pelleted complete cassava feed for growing rabbits. World Rabbit Science, v. 1, n. 4, p. 139-145, 1993.

BERTECHINI A. G. Nutrição de monogástricos. Lavras: UFLA, 2006. 301p.

CHEEKE, P. R. 1994. Nutrition and Nutritional Diseases. In: P. J. Manning, D. H. Ringler and C. E. Newcomer (ed.) The Biology of the Laboratory Rabbit. 2nd ed. Academic Press, New York. 321 p.

DE BLAS J. C.; FRAGA M. J.; RODRÍGUEZ M. Units for feed evaluation requirements for commercially rabbits. Journal of Animal Science, v. 60, p. 1021-1028, 1985.

DE BLAS, J. C., MATEOS, G. G. Feed formulation. In: DE BLAS, C., WISEMAN, J. The nutrition of the rabbit. Cambridge: CAB International, p. 241-253, 1998.

EIBEN C.; GIPPERT T.; GÓDON-SURMANN K.; PODMANICZKY B,; KUSTOS K. Effect of dietary phosphorus reduction and phytase suplementation on growth of the rabbits. In: WORLD RABBIT CONGRESS, 9, 2008, Verona. Proceedings... Verona, 2008. p. 631-636. Disponível em http://world-rabbitscience.com/WRSA-Proceedings/Congress-2008-

Verona/Papers/N-Eiben2.pdf

FALCÃO E CUNHA L.; SABINO I.; CASTRO-SOLLA L.; BRUNO-SOARES A.; FREIRE J. P. Improving the nutritive value of lupin seed for growing rabbits: galactosidade enzymes vs. washing. In: WORLD RABBIT CONGRESS, 9, 2008, Verona. Proceedings... Verona, 2008. p. 661-666. Disponível em: http://worldrabbit-science.com/WRSA-Proceedings/Congress-2008-

Verona/Papers/N-Falcao.pdf

FARIA H. G.; FERREIRA W. M.; SCAPINELLO C.. OLIVEIRA C. E. A. Efeito da utilização de dietas simplificadas, à base de forragem, sobre a digestibilidade e o desempenho de coelhos Nova Zelândia. Revista Brasileira de Zootecnia, v. 37, n. 10, p. 1797-1801, 2008.

FERNANDEZ-CARMONA, J., BERNAT, F., CERVERA, C., PASCUAL, J. J. High lucerne diets for growing rabbits. World Rabbit Science, v. 6, n.2, p.237240, 1998.

FERREIRA W. M.; HERRERA A. D. P. N.; SCAPINELLO C.; FONTES D. O.; MACHADO L. C.; SOUZA J. D. S. Desempenho produtivo de coelhos em crescimento alimentados com dietas simplificadas com base em forragem. In: REUNIÃO ANUAL DA SOCIEDADE BRASILEIRA DE ZOOTECNIA, 43, 2006, João pessoa. Anais..., 2006. Disponível em: http://www.sbz.org.br/reuniaoanual/anais/arq_reuniao_an ual/sbz2006.rar

FERREIRA M.; MACHADO L. C.; FERREIRA W. M.;
SILVA J. Parte aérea de diferentes cultivares de mandioca como fonte de fibra para utilização na alimentação animal. Revista Raízes e Amidos Tropicais, v. 7, n. 1, p. 1-11, 2011.

GIDENNE T. Recent advances in rabbit nutrition: emphasis on fibre requirements, a review. World Rabbit Science, v. 8, n. 1, p. 23-32, 2000.

GUO-XIAN Z.; ZHI-RUA F.; YU-DING W.; YUN-QI L.; GUAN-ZONG L. 2004. The effects of supplemental microbial phytase in dietas on the growth performance and mineral excretion of rabbits. In: WORLD RABBIT CONGRESS, 8, 2004, Puebla. Proceedings... Puebla, 2004. p. 1114-1120. Disponível em: http://world-rabbitscience.com/WRSA-Proceedings/Congress-2004Puebla/Papers/Physiology-Digestion/D-Zhao.pdf

HARRIS D. J.; CHEEKE P. R.; PATTON N. M. Utilization of high alfafa diets by rabbits. Journal of Applied Rabbit Research, v. 4, n. 2, p. 30-33, 1981.

MACHADO L. C.; FERREIRA W. M.; FARIA H. G.; SCAPINELLO, C.; ALVES, C. S. A. Avaliação da dieta simplificada com base em feno de alfafa para coelhas reprodutoras. Veterinária e Zootecnia, v. 14, n. 2, p. 291299, 2007.

MACHADO, L. C.; FERREIRA W. M.; FARIA, H. G.; SCAPINELLO, C. ; ALVES, C. S. A.; OLIVEIRA, C. E. A.; SOUZA, J. D.; PRATES, R. C. Evaluation of the productive performance of growing rabbits of 30 days to slaughter fed with simplified diets with base on forage. In: American Rabbit Congress, 3, 2006, Maringá. Proceedings..., 2006.

MAERTENS L.; PEREZ J. M.; VILLAMIDE M.; CERVERA C. GIDENNE T.; XICCATO G. Nutritive value of raw materials for rabbits: Egran tables 2002. World Rabbit Science, v. 10, n. 4, p. 157-166, 2002.

MULLER P.B. Bioclimatologia aplicada aos animais domésticos. $2^{\mathrm{a}}$ edição. Porto Alegre: Editora Sulina, 1982. $158 \mathrm{p}$.

OLIVEIRA, A.F.G.; SCAPINELLO, C.; MARTINS, E. N.; JOBIM, C. C.; MONTEIRO, A. C.; FIGUEIRA, J. L. Efeito de dietas semi-simplificadas formuladas com subprodutos de mandioca ensilados ou não sobre o desempenho e características de carcaça de coelhos. Acta Scientiarum. Animal Sciences, v. 33, p. 59-64, 2011.

PASCUAL, J. J.; CERVERA, C.; FERNANDEZCARMONA J. A feeding programme for young rabbit does based on lucerne. World Rabbit Science, v. 10, n. 1, p. 7-13, 2002

ROSTAGNO H. S. Tabelas brasileiras para aves e suínos: composição de alimentos e exigências nutricionais. 2 ed. Viçosa: UFV, Departamento de Zootecnia, 2005. $186 \mathrm{p}$.

SCAPINELLO C.; FALCO J. E.; FURLAN A. C.; FARIA H. G. Valor nutritivo do feno da rama de mandioca (Manihot esculenta, Crantz) para coelhos em crescimento. Revista Brasileira de Zootecnia, v. 28, n. 5, 
p. 1063-1067, 1999.

VALENTE S. S.; SANTIAGO G. S.; FERREIRA W. M.; et al. Desempenho de coelhos em crescimento recebendo dietas com suplementação enzimática. Arquivo Brasileiro de Medicina Veterinária e Zootecnia, v. 52, n. 2, p. 173-177, 2000.

VILLAMIDE, M. J., MAERTENS, C., DE BLAS, C., PEREZ, J. M. Feed evaluation. In: DE BLAS, C., WISEMAN, J. The nutrition of the rabbit. Cambridge: CAB International, p. 89-102, 1998.

Protocolado em:19 mar. 2012. Aceito em: 11 jun. 2012. 